\title{
Desmoglein $\mathbf{2}$ is a substrate of kallikrein 7 in pancreatic cancer Vishnu C Ramani, Leah Hennings and Randy S Haun*
}

\author{
Address: Department of Pathology, Winthrop P. Rockefeller Cancer Institute, University of Arkansas for Medical Sciences, Little Rock, Arkansas, \\ USA \\ Email: Vishnu C Ramani - vpramani@uab.edu; Leah Hennings - lhennings@uams.edu; Randy S Haun* - HaunRandyS@uams.edu \\ * Corresponding author
}

Published: 17 December 2008

BMC Cancer 2008, 8:373 doi:10.1 186/147|-2407-8-373

This article is available from: http://www.biomedcentral.com/I47I-2407/8/373

(C) 2008 Ramani et al; licensee BioMed Central Ltd.

This is an Open Access article distributed under the terms of the Creative Commons Attribution License (http://creativecommons.org/licenses/by/2.0), which permits unrestricted use, distribution, and reproduction in any medium, provided the original work is properly cited.
Received: 24 April 2008

Accepted: 17 December 2008

\begin{abstract}
Background: In a previous report we have demonstrated that the chymotryptic-like serine protease kallikrein 7 (KLK7/hK7) is overexpressed in pancreatic cancer. In normal skin, hK7 is thought to participate in skin desquamation by contributing in the degradation of desmosomal components, such as desmogleins. Thus, the ability of hK7 to degrade desmogleins was assessed and the effect of hK7 expression on desmoglein 2 was examined in cultured pancreatic cancer cells.
\end{abstract}

Methods: The expression of Dsgl, Dsg2, and Dsg3 in pancreatic tissues was examined by immunohistochemistry and their expression in two pancreatic cancer cell lines, BxPC-3 and PancI, was determined by western blot analysis. The ability of hK7 to degrade Dsgl and Dsg2 was investigated using in vitro degradation assays. BxPC-3 cells stably transfected to overexpress hK7 were used to examine the effect of hK7 on cell-surface resident Dsg2.

Results: The levels of immunoreactive Dsgl and Dsg2 were reduced in pancreatic adenocarcinomas compared with both normal pancreatic and chronic pancreatitis tissues. Among the desmosomal proteins examined, Dsg2 exhibited robust expression on the surface of BxPC-3 cells. When hK7 was overexpressed in this cell line, there was a significant increase in the amount of soluble Dsg2 released into the culture medium compared with vector-transfected control cells.

Conclusion: A reduction in the amount of the cell adhesion components Dsgl and Dsg2 in pancreatic tumors suggests that loss of these desmosomal proteins may play a role in pancreatic cancer invasion. Using in vitro degradation assays, both Dsgl and Dsg2 could be readily proteolyzed by hK7, which is overexpressed in pancreatic adenocarcinomas. The enforced expression of hK7 in BxPC-3 cells that express significant amounts of Dsg2 resulted in a marked increase in the shedding of soluble Dsg2, which is consistent with the notion that aberrant expression of hK7 in pancreatic tumors may result in diminished cell-cell adhesion and facilitate tumor cell invasion.

\section{Background}

Pancreatic cancer is one of the deadliest of all human cancers, resulting in more than 30,000 deaths per year in the United States alone, and continues to be a major health problem in terms of detection as well as treatment. Pancreatic cancer is highly invasive and is characterized by early metastasis. Tumor invasion and metastasis is a multi-step process involving several key cellular events [1]. Among the many events leading to tumor dissemination and metastasis, loss of intracellular adhesion is one of the earliest events [2]. 
Among the classes of adhesion molecules, desmosomes have been widely recognized and studied for their various roles in cell adhesion, tissue morphogenesis, and cell signaling [3]. Desmosomes, apart from being adhesive intracellular junctions, also act as a membrane anchor for intermediate filaments [4]. The core of the desmosomal adhesive complex primarily consists of desmogleins (Dsg) and desmocollins (Dsc), glycoproteins belonging to the cadherin superfamily of proteins. At least four different isoforms of desmogleins (Dsg1-4) and three different isoforms of desmocollins (Dsc1-3) have been reported thus far. As seen with many other important adhesion molecules, alterations in the expression of various members of desmosomal family of proteins have been observed in different types of cancer [5]. There is, however, a lack of complete understanding regarding the expression of desmosomal proteins in many different types of cancer and the mechanism by which cancer cells may regulate and overcome the adhesion mediated by desmosomal proteins.

One of the most well characterized mechanisms by which tumor cells can overcome adhesion mediated by intercellular adhesive molecules is by up-regulating the expression of various families of proteases that are capable of proteolyzing one or more of these cellular adhesions [69]. Among the various families of proteases, the kallikreins are known to play an important role in many different disease states, including cancer [10-12]. In a previous study, we have reported that kallikrein 7 (KLK7) $\mathrm{hK} 7$ ) is overexpressed in pancreatic adenocarcinomas and enhances pancreatic cancer cell invasion by shedding Ecadherin [13]. Human kallikrein 7 (hK7), originally named stratum corneum chymotryptic enzyme, was initially characterized from extracts of human skin and shown to play an important role in normal skin desquamation by degrading desmogleins and corneodesmosomes $[14,15]$ along with other kallikreins [16]. However, the effects of hK7 expression on desmosomal proteins in any type of cancer, including pancreatic cancer where overexpression of $K L K 7 / \mathrm{hK} 7$ has been clearly established, have not been studied. Herein, we show for the first time that the overall expression levels of desmogleins 1 and 2 are lower in human pancreatic adenocarcinomas compared to chronic pancreatitis and non-malignant pancreatic tissues and that both of these desmosomal proteins are substrates for hK7. Additionally, expression of KLK7 in the human pancreatic adenocarcinoma cell line BxPC-3 significantly increased the amount of soluble desmoglein 2 shed from the cell surface, which correlates with the in vitro degradation data. These results extend the potential roles for the aberrant expression of hK7 observed in pancreatic cancer and points toward a critical role for this protease in aiding cancer invasion via its action on important cellular adhesive molecules like desmogleins.

\section{Methods \\ Immunohistochemistry}

For each antigen examined, formalin-fixed, paraffinembedded tissue blocks from six non-malignant pancreas, six chronic pancreatitis, and six pancreatic adenocarcinoma tissues were prepared for immunohistochemical analysis. Representative hematoxylin and eosin-stained sections from each tissue were evaluated by microscopic analysis. Sections $(4 \mu \mathrm{m})$ were deparaffinized and rehydrated in xylene followed by graded ethanol. Antigen retrieval was performed in a $95^{\circ} \mathrm{C}$ water bath using 10 mM citrate, pH 6.0, for 30 minutes. Endogenous peroxidase activity was quenched by hydrogen peroxide treatment followed by serum-free protein block (DakoCytomation, Carpinteria, CA). Sections were incubated with desmoglein 1, 2, or 3 antibodies (R\&D Systems, Minneapolis, $\mathrm{MN}$ ), diluted 1:75 in antibody diluent (DakoCytomation), overnight at $4^{\circ} \mathrm{C}$. Immunoreactive staining was detected using a DAKO LSAB+ peroxidase system followed by hematoxylin counterstain. Using direct ELISA and western blot analyses to examine the specificity of the desmoglein antibodies, the supplier reports that the Dsg1 antibody shows approximately 5\% crossreactivity with rhDesmoglein-2 and the Dsg2 antibody shows less than $1 \%$ cross-reactivity with rhDesmoglein-1. The acquisition and use of archived, paraffin-embedded human tissue samples in this study were reviewed and approved by the UAMS Human Research Advisory Committee.

\section{Quantitation of immunohistochemical staining}

To numerically analyze the immunohistochemical staining, virtual slides were created from the stained samples after scanning each specimen using an Aperio ScanScope scanning system (Aperio Technologies, Vista, CA). The ScanScope generated true color digital images of each stained sample, which were viewed using Aperio Imagescope v.6.25 software. The algorithm for determining the intensity of membrane-specific staining provided by the manufacturer was optimized and used to calculate the staining intensity and percent target labeled for each sample by digitally analyzing the color intensity. A color markup image for each slide was obtained based on membrane staining intensity. The output was viewed as determinations of staining intensity ranging from $0+$ to $3+$ to correlate with conventional manual scoring methods and the percentage of cells stained for either Dsg1 or Dsg2 was averaged for non-malignant, pancreatitis, pancreatic cancer samples and represented as a stacked column graph.

\section{In vitro degradation assays}

Recombinant, pro-hK7 (100 $\mu \mathrm{g} / \mathrm{mL})$ (R\&D Systems) was proteolytically activated using $10 \mu \mathrm{g} / \mathrm{ml}$ thermolysin (R\&D Systems) and its proteolytic activity was verified using a fluorogenic substrate, ES002 (R\&D Systems), 
according to manufacturer's instructions. Thermolysinactivate hK7 (25 ng) was then added to $100 \mathrm{ng}$ of either recombinant Dsg1 or Dsg2 (R\&D Systems). The mixtures were split into two fractions and thermolysin activity was inhibited in both the fractions with $0.4 \mathrm{mM}$ (final concentration) phosphoramidon (Sigma-Aldrich, St. Louis, $\mathrm{MO})$. As a control, chymostatin was added $(0.28 \mathrm{mM}$ final concentration) to one fraction to inhibit hK7 activity. All the samples were incubated at $37^{\circ} \mathrm{C}$ and at 30 minute intervals an aliquot was removed from each mixture, added to SDS-PAGE sample buffer, heated at $95^{\circ} \mathrm{C}$ for 5 minutes, and stored at $4{ }^{\circ} \mathrm{C}$ for further analysis. At the end of the incubation period, all the samples were separated using 4-12\% gradient Bis-Tris NuPAGE gels (Invitrogen, Carlsbad, CA) and transferred to PVDF membranes. The membranes were blocked with a $5 \%$ non-fat milk solution in Tris-buffered saline, $\mathrm{pH} 7.4$, containing $0.1 \%$ Tween-20 (TTBS) then incubated overnight at $4{ }^{\circ} \mathrm{C}$ with either Dsg1 or Dsg2 antibody (R\&D Systems) diluted 1:1250 with TTBS. After washing with TTBS, the blots were incubated for 1 hour at room temperature with HRP-conjugated polyclonal anti-goat immunoglobulins (DakoCytomation) diluted 1:2000 in TTBS. The blots were washed with TTBS and visualized by chemiluminescence using ECL plus reagent (GE Healthcare, Piscataway, NJ) and a ChemiDoc XRS image documentation system and Quantity One analysis software (Bio-Rad, Hercules, CA).

\section{Immunoblotting for Dsg I and Dsg2 in pancreatic cancer cell lines}

Human pancreatic adenocarcinoma cell lines Panc-1 and BxPC-3 (American Type Culture Collection, Manassas, VA) were grown in $10-\mathrm{cm}$ plates at $37^{\circ} \mathrm{C}$ in a $5 \% \mathrm{CO}_{2}$ incubator in Dulbecco's modified Eagle's medium (DMEM) (Invitrogen) containing 10\% fetal bovine serum (Atlanta Biologicals, Norcross, GA). Confluent monolayers were harvested by scraping, washed twice with phosphate-buffered saline (PBS), and lysed with RIPA buffer (1 mM EDTA, $1 \%$ NP- $40,0.5 \%$ deoxycholate, $0.1 \%$ SDS in PBS) containing Complete protease inhibitor cocktail (Roche, Indianapolis, IN). The cell lysates were sonicated on ice and centrifuged at 13,000 rpm to remove any cell debris and the protein concentration of each lysate was determined using a bicinchoninic acid assay (BCA) (Sigma-Aldrich). Two samples of each lysate (50 $\mu \mathrm{g}$ of total protein) were resolved by SDS-PAGE and transferred to PVDF membrane. The membrane was cut and probed for Dsg1 or Dsg2 (upper portion) and GAPDH (lower portion) by western blot.

\section{Immunocytochemistry}

Panc- 1 and BxPC-3 cells $\left(3 \times 10^{4}\right)$ were seeded in Lab-Tek II four-chamber glass slides (Nalge Nunc, Naperville, IL). Confluent monolayers were washed twice with PBS then fixed with $4 \%$ paraformaldehyde in PBS at room temperature for 20 minutes. The fixed cells were washed with PBS, permeabilized with $-20^{\circ} \mathrm{C}$ chilled methanol, washed with PBS, and incubated with serum-free protein block (DAKO). The cells were then incubated overnight at $4{ }^{\circ} \mathrm{C}$ with a polyclonal Dsg2 antibody (diluted 1:75), washed with PBS containing $0.1 \%$ Triton X-100 (TPBS), and incubated with a fluorescein isothiocyanate (FITC)-conjugated rabbit anti-goat antibody (DakoCytomation) for 2 hours at room temperature. Cells were washed with TPBS, mounted with Vectashield mounting medium (Vector Laboratories, Burlingame, CA), and visualized using an Olympus BX41 fluorescent microscope (Olympus USA, Melville, NY). Images were captured with a SPOT-RT digital camera and software (Diagnostic Instruments, Sterling Heights, MI).

\section{Detection of soluble desmoglein 2}

One million vector-transfected or KLK7-expressing BxPC3 cells [17] were seeded in 10-cm dishes. Growth medium from confluent monolayers was removed, cells were washed twice with serum-free medium (SFM), and then incubated in SFM containing $1.5 \mu \mathrm{g} / \mathrm{ml}$ puromycin for 24 hours. The conditioned medium was removed and centrifuged at $1500 \mathrm{rpm}$ for 5 minutes at $4^{\circ} \mathrm{C}$ to remove detached cells and debris and then concentrated using Amicon Ultra-4 centrifugal filter units, $10-\mathrm{kDa}$ nominal molecular weight limit (Millipore, Billerica, MA), according to manufacturer's instructions. The cell monolayers were harvested by trypsinization and single-cell suspensions were prepared and enumerated using a Z1 Coulter counter (Beckman Coulter, Fullerton, CA). The total number of cells per volume of final concentrate for each sample was calculated and the volume of concentrate corresponding to $10^{6}$ cells for each sample was mixed with SDS sample buffer and heated at $95^{\circ} \mathrm{C}$ for 5 minutes. The samples were then separated using a NuPage $4-12 \%$ gradient polyacrylamide gel (Invitrogen) and transferred to a PVDF membrane. The immunoblot was blocked with a $5 \%$ non-fat milk solution in TTBS then incubated overnight with anti-Dsg2 polyclonal antibody (diluted $1: 1250)$ (R\&D Systems). After washing with TTBS, the blot was incubated with horseradish peroxidase-(HRP) conjugated anti-goat immunoglobulins (diluted 1:2000) (DakoCytomation) and soluble Dsg2 (sDsg2) was visualized by chemiluminescence using ECL plus reagent and a ChemiDoc XRS image documentation system. The amount of sDsg2 released into the conditioned medium of each sample was quantified using Quantity One image analysis software. The mean intensity of three independent experiments was determined and analyzed for statistical significance using an unpaired, 2-tailed $t$-test (GraphPad Software, San Diego, CA). 


\section{Results \\ Intensity of membrane staining for desmosomal proteins Dsg I and Dsg2 is lower in human pancreatic cancer compared to normal pancreas and chronic pancreatitis}

Six independent sections of normal human pancreas, chronic pancreatitis, or pancreatic adenocarcinoma tissue were stained for desmogleins 1, 2, and 3. Desmogleins 1 and 2 were clearly present in all of the normal pancreatic tissue samples examined and showed intense staining at the cell-cell borders (Fig. 1A and 2A, respectively). In contrast, Dsg3 was not detected in any of the pancreatic tissues (data not shown). The chronic pancreatitis samples showed staining intensity and distribution similar to the normal pancreatic samples for Dsg1 (Fig. 1B) and Dsg2 (Fig. 2B). All the pancreatic cancer samples displayed an intense desmoplastic response, the synthesis and deposition of collagenous material by stromal myofibroblasts surrounding the adenocarcinoma, and the membrane staining for both Dsg1 (Fig. 1C) and Dsg2 (Fig. 2C) was distinctly weaker in most of the cancer samples analyzed. The correspondence between increased hK7 expression in pancreatic tumors and loss of Dsg2 is highlighted in the immunohistochemistry studies we have performed. For example, the tumor section stained for Dsg2 (Figure 2C, this study), showing a loss of Dsg2 immunoreactivity, is from the same tissue used to demonstrate increased hK7 expression in pancreatic tumors (Figure 2A, ref. [13]).

To further quantify the intensity of membrane staining for Dsg1 and Dsg2 in the different tissue samples, the stained slides were scanned with an Aperio ScanScope and areas of cellularity in the digital images were selected by a pathologist (L.H.) and processed to calculate the staining intensity and percent label for each sample. The average percentage of cells stained for Dsg1 within each intensity category (e.g., 1+, 2+) was nearly equal between the normal pancreatic tissues and chronic pancreatitis samples (Fig. 1D). The pancreatic cancer samples, however, showed an almost complete loss of cells displaying intense (3+) Dsg1 staining and a marked decrease in cells with $2+$ intensity with a concomitant increase in cells with weak $(1+)$ or absent $(0+)$ Dsg1 staining.

An examination of Dsg2 immunoreactivity revealed that the most intense staining $(3+)$ was observed in the normal pancreatic tissues $(28.4 \%)$, although the chronic pancreatitis samples $(11.2 \%)$ still showed a higher average percentage of cells with $3+$ intensity compared to the pancreatic adenocarcinoma samples (4.3\%) (Fig. 2D). The average number of cells with $2+$ intensity of staining was nearly equal in the normal and chronic pancreatitis samples and somewhat higher in pancreatic cancer samples, while the number of cells with $(1+)$ intensity was higher in chronic pancreatitis $(47.8 \%)$ and pancreatic cancer $(47.7 \%)$ samples compared with the normal pan-
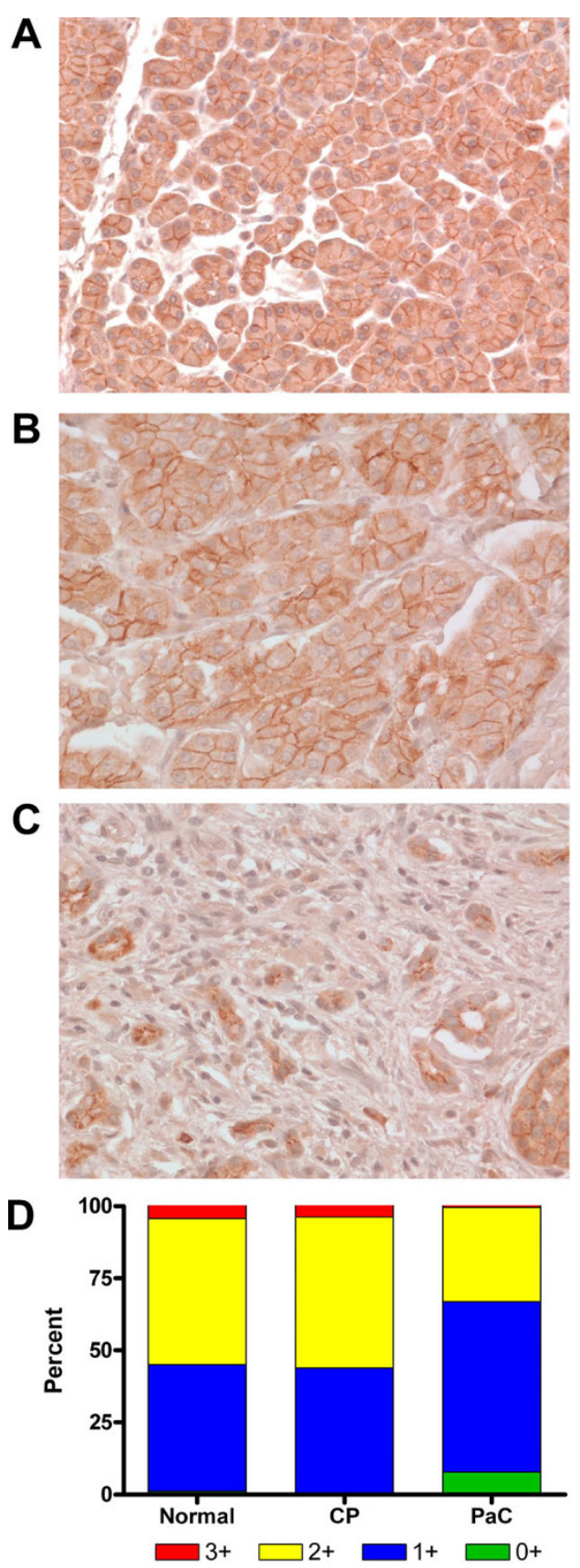

\section{Figure I}

Desmoglein I is present in lower levels in pancreatic cancer compared to normal and chronic pancreatitis tissue. Representative pancreatic tissue sections stained for Dsgl showed high membrane staining in (A) normal pancreas, $(B)$ chronic pancreatitis (CP), and (C) weaker staining in pancreatic adenocarcinoma $(\mathrm{PaC})$ samples. Original magnification $\times 400$. (D) Staining intensity of the cell membranes was quantitated (see Methods), categorized into intensity ranges from $0+$ to $3+$, and the average percentage of cells in each group was determined and represented in a stacked graph for each tissue type $(n=6)$. 
A

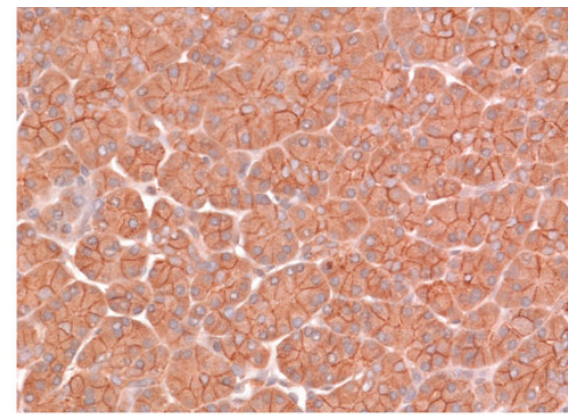

B

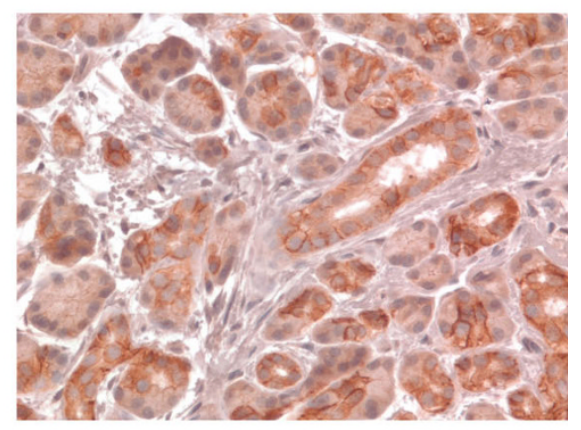

C

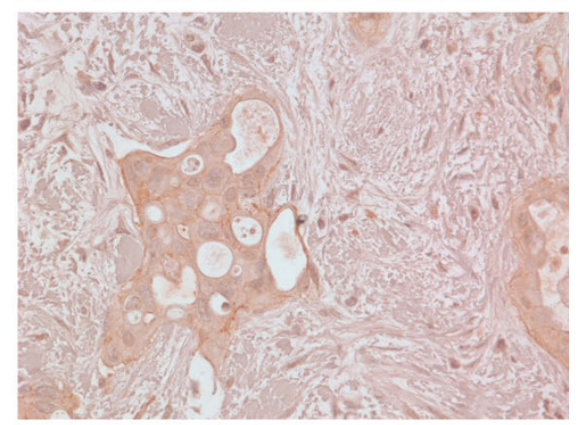

D

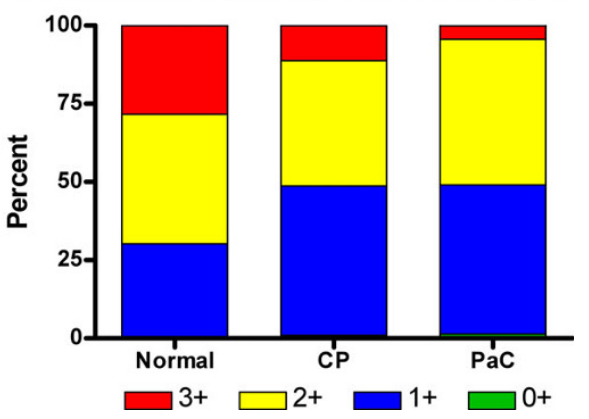

Figure 2

Desmoglein 2 staining is lower in pancreatic cancer cell membranes compared to normal and chronic pancreatitis tissue. Representative pancreatic tissue sections stained for Dsg2 showed high levels of membrane staining in (A) normal pancreas and (B) chronic pancreatitis (CP) samples. Membrane staining was much weaker in (C) pancreatic adenocarcinoma $(\mathrm{PaC})$ samples. Original magnification $x 400$. (D) Staining intensity of the cell membranes was quantitated (see Methods), categorized into intensity ranges from $0+$ to $3+$, and the average percentage of cells in each group was determined and represented in a stacked graph for each tissue type $(n=6)$. creatic tissues (30\%). Although the percentage of cells lacking demonstrable Dsg2 staining $(0+)$ increased with the severity of disease (i.e., pancreatic cancer $>$ chronic pancreatitis > normal pancreas), the numbers were modest in all three tissue types. (Fig. 2D). Thus, overall the average intensity of membranous desmoglein staining was decreased in adenocarcinomas compared to normal pancreas and pancreatitis samples.

\section{hK7 cleaves both recombinant Dsg I and Dsg2 in vitro}

To determine whether hK7 could participate in decreasing the levels of Dsg1 and Dsg2 through proteolytic cleavage, in vitro degradation assays were performed using recombinant hK7 and desmosomal proteins. Upon incubation with thermolysin-activated hK7, recombinant Dsg1 was rapidly degraded with little detectable protein remaining after 60 minutes (Fig. 3A, left). Recombinant Dsg2 was also cleaved by hK7 in a time-dependent manner, however, in contrast to the complete proteolysis of Dsg1, the cleavage of Dsg2 resulted in distinct proteolytic fragments (Fig. 3B, left). All of the degradation assays were performed in the presence of phosphoramidon, which inhibits the thermolysin used to activate hK7. To verify that the degradation of the desmogleins was due to hK7 activity and not due to any residual activity of thermolysin, a parallel set of assays was performed that included chymostatin, an oligopeptide that inhibits serine proteases with chymotrypsin-like substrate specificity (Figs. $3 \mathrm{~A}$ and $3 \mathrm{~B}$, right). Proteolysis of both the desmosomal proteins by hK7 was effectively inhibited in the presence of chymostatin.

\section{Levels of desmogleins in human pancreatic cancer cells}

To identify pancreatic cancer cell lines that could be used to examine hK7-dependent cleavage of desmosomal proteins in a cell-based system, cell lysates were prepared from Panc- 1 and BxPC-3 cell lines and assessed for the levels of Dsg1 and Dsg2 by western blot analysis. Dsg1 was not detected in either of the cell lines (Fig. 4A, lanes 1 and 2 ), whereas Dsg2 was readily detected in BxPC-3 but not Panc-1 cell lysates (Fig. 4A, lanes 3 and 4, respectively). Due to the significant expression of Dsg2 in BxPC-3 cell lysates, immunocytochemical staining was performed on intact cells to determine its cellular localization and distribution. The staining revealed a robust cell-cell border distribution similar to the pattern seen in epithelial tissues (Fig. 4B).

\section{Effect of hK7 expression on Dsg2 in BxPC-3 pancreatic cancer cells}

Since BxPC-3 cells exhibit significant amounts of Dsg2, but do not express hK7 (data not shown), these cells were utilized to investigate the effects of enforced hK7 expression on Dsg2 in a cell-based system. BxPC-3 cells were transfected with a KLK7-expression construct or empty 


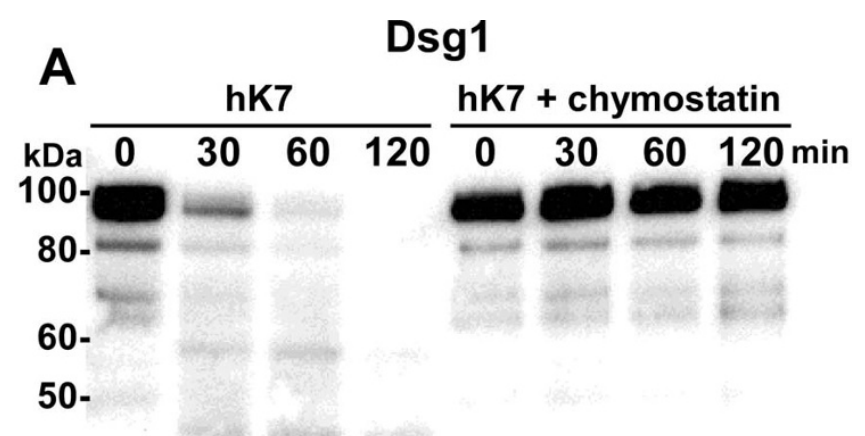

$\begin{array}{llllllll}1 & 2 & 3 & 4 & 5 & 6 & 7 & 8\end{array}$

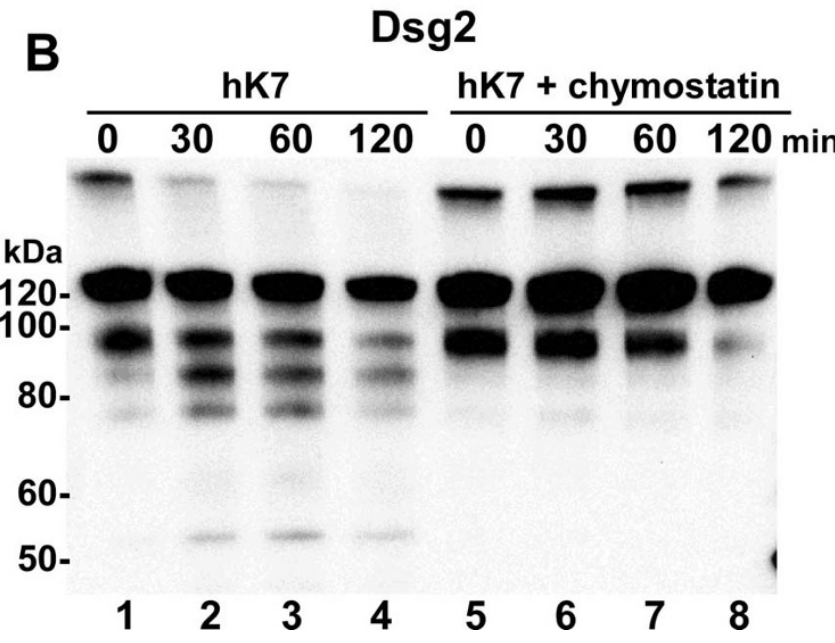

Figure 3

Desmoglein I and 2 are substrates for hK7 in vitro. Thermolysin-activated hK7 was incubated with recombinant Dsgl (A) or Dsg2 (B) for the indicated times in the absence (lanes I-4) or presence (lanes 5-8) of the hK7 inhibitor chymostatin. Reaction products were separated by SDS-PAGE and visualized by western blot using either anti-Dsg I or antiDsg2 antibodies. Sizes of protein markers are indicated on the left.

vector and stable transfectants were generated [17]. To obviate clonal effects, two independent KLK7-expressing clones, BxPC-3/hK7 clone\#3 and BxPC-3/hK7 clone\#7, were analyzed and compared to a vector-transfected control clone, BxPC-3/Vec. To examine the cleavage of Dsg2 from the cell surface in the presence of hK7, concentrated conditioned medium from hK7-expressing and vectortransfected control cells was prepared and the level of soluble Dsg2 was determined by western blot. Both of the hK7-expressing clones examined displayed higher levels of an $80-\mathrm{kDa}$ soluble Dsg2 product compared to the vector-transfected clone (Fig. 5); thus supporting the notion that aberrant expression of hK7 may lead to the cleavage of Dsg2 from the cell surface.
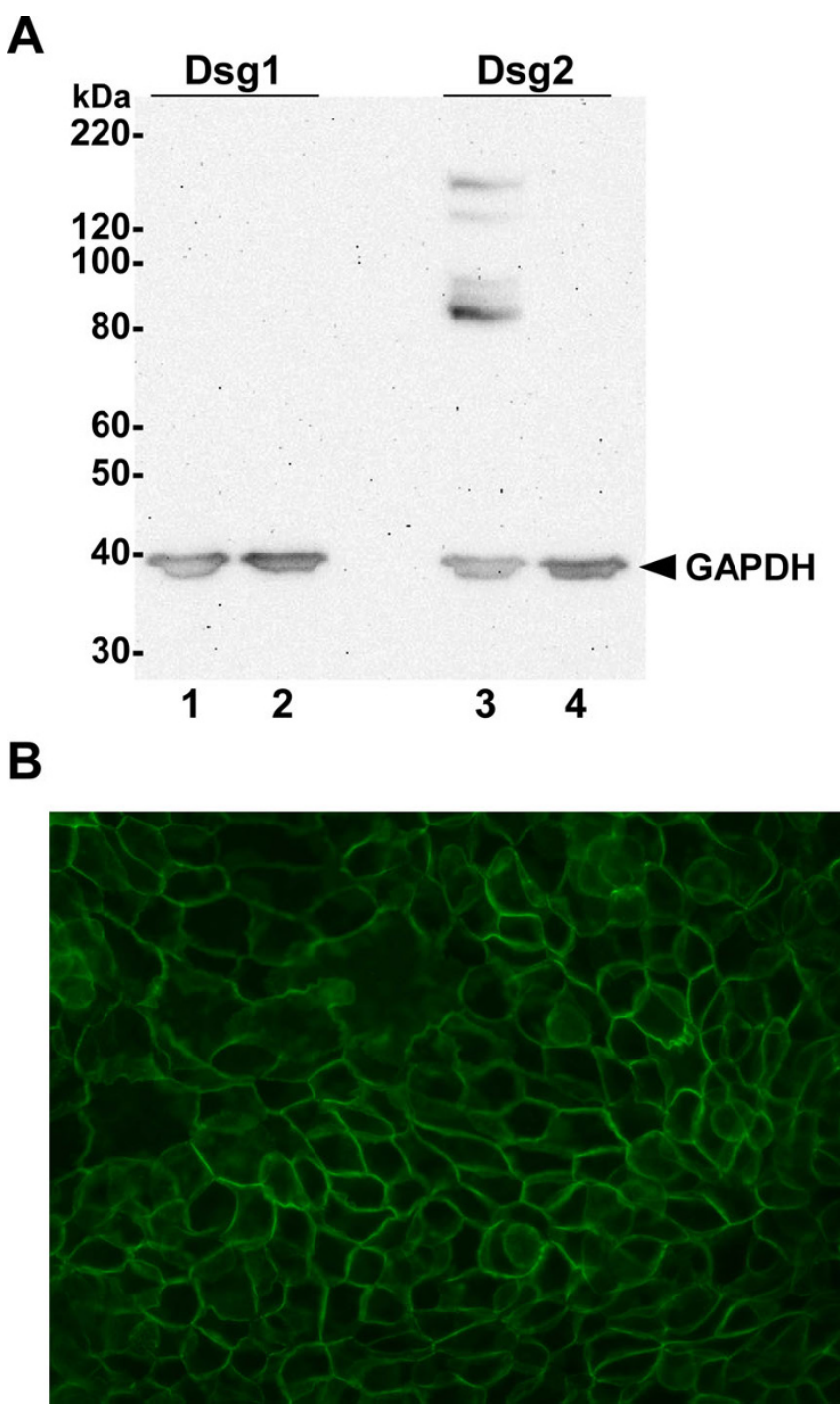

Figure 4

Expression of desmogleins in human pancreatic adenocarcinoma cell lines. (A) Equal amounts of total protein from whole cell lysates of human pancreatic cancer cell lines BxPC-3 (lanes I and 3) and Panc-I (lanes 2 and 4) were separated by SDS-PAGE and visualized by western blot using antiDsgl and anti-Dsg2 antibodies, as indicated. GAPDH levels were monitored as a loading control (arrowhead). Sizes of protein markers are indicated on the left. Appreciable levels of Dsg2 were detected in BxPC-3 cells, but not in Panc-I cells. Dsg I was not detected in either of the cell lines. (B) Immunocytochemistry of Dsg2 in BxPC-3 cells using a FITCconjugated secondary antibody showed specific cell membrane localization. Original magnification $\times 400$.

\section{Discussion}

Cell-cell adhesion and cell-extracellular matrix adhesion play a critical role as limiting factors during the early steps of invasion $[1,18,19]$. Various classes of cell adhesion 
molecules, including desmosomes, are affected in different types of cancer and tumor cells can regulate the expression and function of these adhesive molecules via various mechanisms $[20,21]$. Components that comprise these intercellular junctions play a critical role in determining the function and strength of these adhesive complexes [22]. Desmogleins and desmocollins, along with plakoglobin, desmoplakin, and plakophilin, represent the
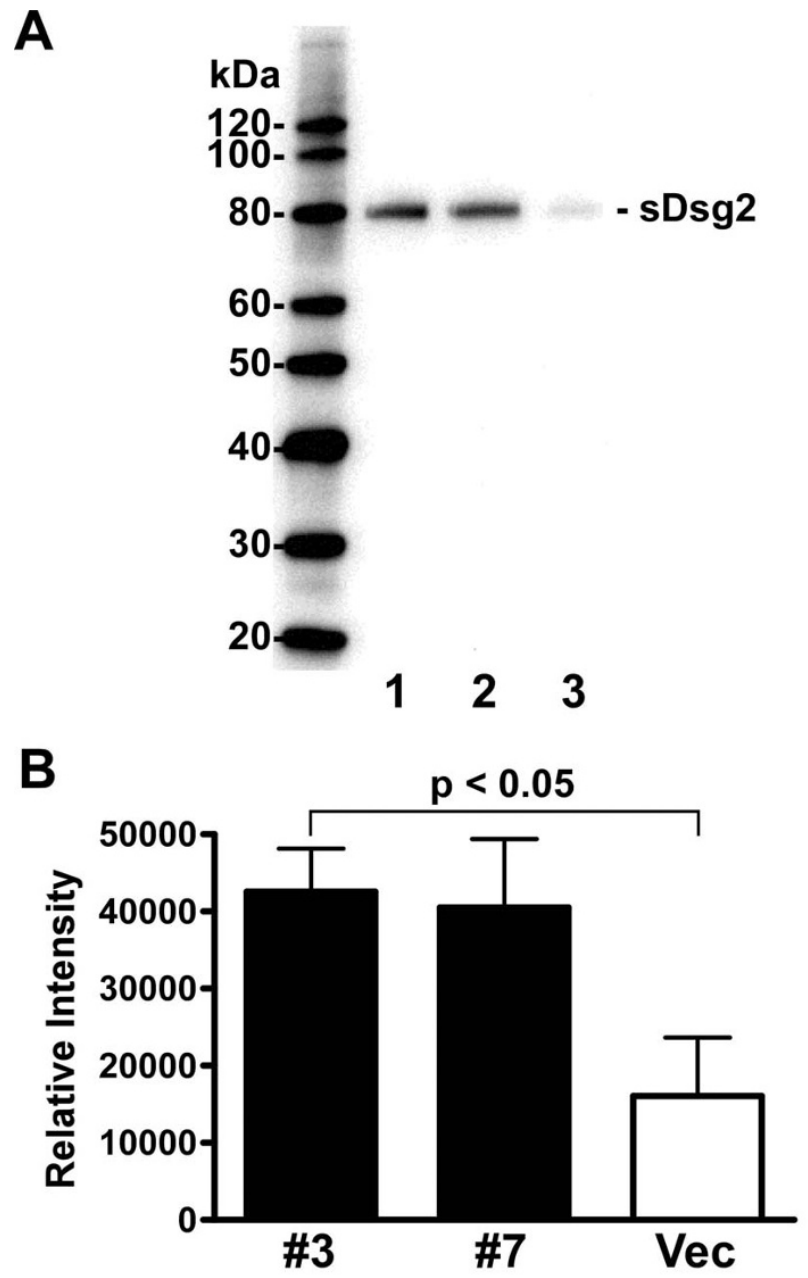

Figure 5

Levels of soluble Dsg2 in conditioned medium of KLK7-expressing BxPC-3 cells are higher than in vector-transfected control cells. (A) Levels of soluble Dsg2 (sDsg2) in concentrated conditioned medium from hK7expressing clones BxPC-3/hK7 clone\#3 (lane I) and clone\#7 (lane 2) and vector-transfected clone BxPC-3/Vec (lane 3) were determined by western blot. Sizes of protein markers are indicated on the left. (B) The relative amount of the 80$\mathrm{kDa}$ sDsg2 immunoreactive product was determined for KLK7-transfected (solid bars) and vector-transfected (open bar) BxPC-3 cells by densitometric analysis using Quantity One software and the average relative intensity is depicted. Vertical bars represent SEM $(n=3)$. essential and minimum number of components that can form an intact functional desmosome [23,24]. Desmogleins, which form an integral part of the desmosomal adhesive core, are affected in many types of cancer. Various desmoglein proteins have been shown to be downregulated in urothelial carcinoma [25], oral squamous cell carcinoma $[26,27]$, esophageal squamous cell carcinoma [28], and squamous intraepithelial lesions of the cervix [29]. Desmosomal constituents, like Dsg2, have been demonstrated to be expressed abnormally in gastric cancer [30] and its decreased expression has been associated with poorer prognosis for diffuse-type gastric cancer [31]. However, a clear understanding of the expression levels of desmogleins and the possible mechanisms leading to their decreased expression in other types of cancer, like pancreatic cancer, is still lacking.

Kallikrein 7, a chymotryptic-like serine protease that is prevalent in the stratum corneum, has been shown to directly cleave corneodesmosomes and desmosomes in vitro [32]; thus implicating its participation in desquamation in normal human skin [33]. Studies have also shown that hK7 is overexpressed in squamous cervical cancerous cells [34], ovarian tumor cells $[35,36]$, cervical adenocarcinomas [37], and in lung cancer [38]. Moreover, recently we have further demonstrated that $K L K 7 / \mathrm{hK} 7$ is overexpressed in pancreatic adenocarcinomas [13]. However, the ability of hK7 expressed in any of these cancers to act upon desmosomal proteins, like desmogleins, which are substrates for hK7 in normal physiology, has never been explored.

Based on the aberrant expression of hK7 in pancreatic cancer, in this study we sought to determine whether the desmogleins may be substrates of hK7 in pancreatic cancer. The expression and distribution of individual desmosomal cadherins is tissue specific [39]. For example, Dsg2 is ubiquitously expressed in all tissues containing desmosomes [40], whereas Dsg1 and Dsg3 are mainly restricted to stratified squamous epithelia [41]. In the epidermis, Dsg1 is expressed throughout the upper differentiating cell layers, while Dsg3 is restricted to basal keratinocytes [41]. Thus, as a positive control we stained sections of normal human skin with the Dsg1 antibody used to detect its expression in pancreatic tissues (Additional file 1). Consistent with previous findings [42], Dsg1 was detected throughout the epidermis from superficial to basal layers. In recent studies in mice, the presence of DSG1 and DSG3 mRNAs have been reported in many epithelial organs [41]. As a first step, we surveyed human pancreatic tissue samples representing non-malignant, chronic pancreatitis, and pancreatic cancer specimens for the expression of desmoglein proteins by immunohistochemistry. Upon analysis, both desmogleins 1 and 2 showed intense membrane staining in normal pancreas and chronic pancreati- 
tis samples. The intensity of staining for both these desmosomal proteins, however, was qualitatively much weaker in pancreatic cancer tissues. To quantitate the intensity of the membrane staining in a more objective manner, the stained sections were scanned and the digital images processed to produce a numerical representation of the staining intensity and stratification into groups typically reported in a manual analysis by a pathologist (e.g., $3+$ staining). This analysis revealed that the average percentage of cells within each intensity group was similar for Dsg1 between the non-malignant and chronic pancreatitis samples, but that the percentage of more intensely stained cells for Dsg1 decreased markedly in the pancreatic cancer samples. In contrast, with Dsg2 the average number of cells with intense staining (3+) was markedly higher in non-malignant pancreas samples compared to either of the other two diseased conditions. With both Dsg1 and Dsg2, there was a pronounced shift toward the lower intensity categories in the pancreatic cancer samples. Thus, there appeared to be a clear inverse correlation between the expression of hK7 and the membrane intensity of desmosomal proteins in pancreatic samples from diseased and non-diseased conditions. No appreciable Dsg3 immunoreactivity could be detected in any of the pancreatic tissue samples. Since the ability of the antibodies used to detect Dsg3 by immunohistochemistry was verified using sections of normal human skin as a positive control (data not shown), these findings indicate that Dsg3 is not expressed at detectable levels in human pancreas.

To provide more direct evidence for a relationship between hK7 expression and the decreased intensity of desmogleins observed by immunohistochemistry, in vitro substrate degradation assays were performed. In support of an association between hK7 and the desmogleins, hK7 was able to proteolyze both Dsg1 and Dsg2. Proteolysis of Dsg1 resulted in a rapid degradation of recombinant Dsg1 into fragments undetectable by western blot. Interestingly, incubation of Dsg2 with hK7 resulted in cleavage of Dsg2 into distinct fragments rather than indiscriminant degradation. As further evidence of the specificity of hK7, we have tested other potential substrates under similar conditions. For example, when laminin was incubated with activated hK7, no significant cleavage was observed even after 24 hours (Additional file 2); thus, not all proteins are subject to hK7-directed proteolysis.

To identify a pancreatic cancer cell-based model to further examine the proteolysis of desmogleins by hK7, cell lysates from two pancreatic cancer cell lines, BxPC-3 and Panc-1, were screened for Dsg1 and Dsg2 by western blot analysis. This revealed that only Dsg2 was expressed in appreciable levels in BxPC-3 cells. Since BxPC-3 cells do not express hK7, we used these cells to mimic the aberrant expression of KLK7 observed in pancreatic tumors by expressing high levels of hK7 through stable transfection. In this manner, the effects on Dsg2 in cells with and without KLK7 expression could be compared, which could yield insights into changes in desmosomal proteins upon hK7 expression. In BxPC-3 cells expressing hK7, a marked increase in the shedding of an 80-kDa sDsg2 fragment was observed compared to a vector-transfected control. These findings suggest that decreased levels of desmosomal proteins seen in pancreatic cancer may result from the action of hK7 overexpressed in these tumors.

A comparison of KLK7-transfected and vector-transfected pancreatic cancer cells reveals that hK7 expression alters cell morphology (Additional file 3), with cells expressing hK7 exhibiting a more flattened, less refractive appearance and reduced cell-cell contacts. Using in vitro assays, we have demonstrated that hK7 can cleave both E-cadherin [13] and desmogleins (this report) independently. In cellbased assays where both E-cadherin and desmogleins (Dsg2) are present, we can not dissect whether the action of hK7 upon a particular substrate is a primary or secondary effect, or whether both substrates are acted upon concurrently. Based upon our in vitro results, we favor the notion that hK7 cleaves each substrate from the cell surface independently, with their combined loss contributing to the morphological changes observed. Other hK7 substrates that have yet to be elucidated, however, may also participate in this process.

\section{Conclusion}

In this report we have shown that the levels of cell-surface resident Dsg1 and Dsg2 are reduced in pancreatic adenocarcinomas compared with normal and chronic pancreatitis tissues. We have also demonstrated that these desmosomal proteins are substrates of the chymotrypticlike protease $\mathrm{hK} 7$, which is overexpressed in pancreatic cancer. Using a cell-based system, we have found that expression of hK7 results in a marked increase in the shedding of an $80-\mathrm{kDa}$ soluble fragment of Dsg2 into the conditioned medium of BxPC-3 cells. These results, therefore, provide evidence that hK7 may play a critical role in the invasion of cancer cells in which it is aberrantly expressed by degrading important cell adhesive molecules like desmogleins and E-cadherin [13].

\section{Competing interests}

The authors declare that they have no competing interests.

\section{Authors' contributions}

VCR participated in the design of the study, carried out all of the technical procedures, and performed the statistical analyses. LH performed the immunohistochemical analyses. RH supervised the design and implementation of the study. All authors participated in the preparation of the manuscript and have read and approved the final manuscript. 


\section{Additional material}

\section{Additional file 1}

Desmoglein 1 staining in normal human skin. As a positive control, immunohistochemistry performed on sections of normal human skin with the Dsg1 antibody used for staining pancreatic tissues revealed basilar and suprabasilar epidermal staining.

Click here for file

[http://www.biomedcentral.com/content/supplementary/14712407-8-373-S1.png]

\section{Additional file 2}

Laminin is not a substrate of $h K 7$ in vitro. Thermolysin-activated $h K 7$ (200 ng) was incubated with $1 \mu \mathrm{g}$ of laminin for the indicated times and the reaction products were separated by SDS-PAGE and visualized by Coomassie staining. Sizes of protein markers are indicated on the left. Click here for file

[http://www.biomedcentral.com/content/supplementary/14712407-8-373-S2.png]

\section{Additional file 3}

Expression of $h K 7$ in BxPC-3 cells results in an altered cell morphology. Phase-contrast (upper) or rhodamine phalloidin-stained (lower) images of $h K 7$-expressing clones BxPC-3/hK7 clone\#3 and clone\#7 reveal altered cell morphology compared with vector-transfected cells (BxPC-3/ $V e c)$. Cells expressing $h K 7$ display reduced cell-cell contacts and a more flattened, less refractive appearance.

Click here for file

[http://www.biomedcentral.com/content/supplementary/14712407-8-373-S3.png]

\section{Acknowledgements}

This research was supported by funds, in part, from the UAMS Graduate Student Research Fund (VCR).

\section{References}

I. Hanahan D, Weinberg RA: The hallmarks of cancer. Cell 2000, I 00(I):57-70.

2. Zetter BR: Adhesion molecules in tumor metastasis. Semin Cancer Biol 1993, 4(4):219-229.

3. Green KJ, Gaudry CA: Are desmosomes more than tethers for intermediate filaments? Nat Rev Mol Cell Biol 2000, I (3):208-2I6

4. Kowalczyk AP, Bornslaeger EA, Norvell SM, Palka HL, Green KJ Desmosomes: intercellular adhesive junctions specialized for attachment of intermediate filaments. Int Rev Cytol 1999, 1 85:237-302.

5. Chidgey M, Dawson C: Desmosomes: a role in cancer? $\mathrm{Br} / \mathrm{Can}$ cer 2007, 96(12): 1783-1787.

6. Bussemakers MJ, Schalken JA: The role of cell adhesion molecules and proteases in tumor invasion and metastasis. World J Urol 1996, I4(3): I51-156.

7. Monsky WL, Chen WT: Proteases of cell adhesion proteins in cancer. Semin Cancer Biol I993, 4(4):25I-258.

8. Netzel-Arnett S, Hooper JD, Szabo R, Madison EL, Quigley JP, Bugge $\mathrm{TH}$, Antalis TM: Membrane anchored serine proteases: a rapidly expanding group of cell surface proteolytic enzymes with potential roles in cancer. Cancer Metastasis Rev 2003, 22(23):237-258.

9. Stetler-Stevenson WG, Yu AE: Proteases in invasion: matrix metalloproteinases. Semin Cancer Biol 200 I, I I (2): | 43-I 52.

10. Borgono CA, Diamandis EP: The emerging roles of human tissue kallikreins in cancer. Nat Rev Cancer 2004, 4(I I ):876-890.

II. Diamandis EP, Yousef GM: Human tissue kallikreins: a family of new cancer biomarkers. Clin Chem 2002, 48(8): I I 98-I 205.
12. Diamandis EP, Yousef GM, Luo LY, Magklara A, Obiezu CV: The new human kallikrein gene family: implications in carcinogenesis. Trends Endocrinol Metab 2000, I I (2):54-60.

13. Johnson SK, Ramani VC, Hennings L, Haun RS: Kallikrein 7 enhances pancreatic cancer cell invasion by shedding E-cadherin. Cancer 2007, I09(9): |8| |- |820.

14. Descargues P, Deraison C, Prost C, Fraitag S, Mazereeuw-Hautier J, D'Alessio M, Ishida-Yamamoto A, Bodemer C, Zambruno G, Hovnanian A: Corneodesmosomal cadherins are preferential targets of stratum corneum trypsin- and chymotrypsin-like hyperactivity in Netherton syndrome. J Invest Dermatol 2006, I 26(7): I622-1632

I5. Egelrud T, Lundstrom A: A chymotrypsin-like proteinase that may be involved in desquamation in plantar stratum corneum. Arch Dermatol Res 199I, 283(2): 108-II2.

16. Borgono CA, Michael IP, Komatsu N, Jayakumar A, Kapadia R, Clayman GL, Sotiropoulou G, Diamandis EP: A potential role for multiple tissue kallikrein serine proteases in epidermal desquamation. J Biol Chem 2007, 282(6):3640-3652.

17. Ramani VC, Haun RS: The extracellular matrix protein fibronectin is a substrate for kallikrein 7. Biochem Biophys Res Commun 2008, 369(4): I I69-II73.

18. Behrens ]: The role of cell adhesion molecules in cancer invasion and metastasis. Breast Cancer Res Treat I993, 24(3): I75-I84.

19. Cavallaro $U$, Christofori $G$ : Cell adhesion in tumor invasion and metastasis: loss of the glue is not enough. Biochim Biophys Acta 200I, I 552(I):39-45

20. Hajra KM, Fearon ER: Cadherin and catenin alterations in human cancer. Genes Chromosomes Cancer 2002, 34(3):255-268.

21. Yoshiura K, Kanai Y, Ochiai A, Shimoyama Y, Sugimura T, Hirohashi $S$ : Silencing of the E-cadherin invasion-suppressor gene by CpG methylation in human carcinomas. Proc Natl Acad Sci USA 1995, 92(1 6):7416-7419.

22. Schwarz MA, Owaribe K, Kartenbeck J, Franke WW: Desmosomes and hemidesmosomes: constitutive molecular components. Annu Rev Cell Biol 1990, 6:461-49I.

23. Chitaev NA, Troyanovsky SM: Direct Ca2+-dependent heterophilic interaction between desmosomal cadherins, desmoglein and desmocollin, contributes to cell-cell adhesion. J Cell Biol I997, I38(I):|93-20|.

24. Marcozzi C, Burdett ID, Buxton RS, Magee Al: Coexpression of both types of desmosomal cadherin and plakoglobin confers strong intercellular adhesion. J Cell Sci 1998, I I I (Pt 4):495-509.

25. Conn IG, Vilela MJ, Garrod DR, Crocker J, Wallace DM: Immunohistochemical staining with monoclonal antibody 32-2B to desmosomal glycoprotein $I$. Its role in the histological assessment of urothelial carcinomas. $\mathrm{Br} J$ Urol 1990 , 65(2): $176-180$.

26. Hiraki A, Shinohara M, Ikebe T, Nakamura S, Kurahara S, Garrod DR: Immunohistochemical staining of desmosomal components in oral squamous cell carcinomas and its association with tumour behaviour. Br J Cancer | 996, 73( I 2): | 49 | - | 497.

27. Imai K, Kumagai S, Nakagawa K, Yamamoto E, Nakanishi I, Okada Y: Immunolocalization of desmoglein and intermediate filaments in human oral squamous cell carcinomas. Head Neck 1995, I 7(3):204-212.

28. Pittella F, Katsube K, Takemura T, Hashimoto T, Kawano T, Garrod $D$, Takagi M: Perinuclear and cytoplasmic distribution of desmoglein in esophageal squamous cell carcinomas. Pathol Res Pract 200I, 197(2):85-91.

29. Alazawi WO, Morris LS, Stanley MA, Garrod DR, Coleman N: Altered expression of desmosomal components in highgrade squamous intraepithelial lesions of the cervix. Virchows Arch 2003, 443(I):51-56.

30. Biedermann K, Vogelsang $H$, Becker I, Plaschke S, Siewert JR, Hofler $H$, Keller G: Desmoglein 2 is expressed abnormally rather than mutated in familial and sporadic gastric cancer. J Pathol 2005, 207(2): 199-206

31. Yashiro M, Nishioka N, Hirakawa K: Decreased expression of the adhesion molecule desmoglein-2 is associated with diffusetype gastric carcinoma. Eur J Cancer 2006, 42( I 4):2397-2403

32. Caubet C, Jonca N, Brattsand M, Guerrin M, Bernard D, Schmidt R, Egelrud T, Simon M, Serre G: Degradation of corneodesmosome proteins by two serine proteases of the kallikrein family, SCTE/KLK5/hK5 and SCCE/KLK7/hK7. I Invest Dermatol 2004, I 22(5): I 235-1244. 
33. Suzuki $Y$, Koyama J, Moro O, Horii I, Kikuchi K, Tanida M, Tagami H: The role of two endogenous proteases of the stratum corneum in degradation of desmoglein- $I$ and their reduced activity in the skin of ichthyotic patients. BrJ Dermatol 1996, 134(3):460-464.

34. Santin AD, Cane S, Bellone S, Bignotti E, Palmieri M, De Las Casas LE, Roman JJ, Anfossi S, O'Brien T, Pecorelli S: The serine protease stratum corneum chymotryptic enzyme (kallikrein 7) is highly overexpressed in squamous cervical cancer cells. Gynecol Oncol 2004, 94(2):283-288.

35. Shigemasa K, Tanimoto H, Underwood LJ, Parmley TH, Arihiro K, Ohama K, O'Brien T]: Expression of the protease inhibitor antileukoprotease and the serine protease stratum corneum chymotryptic enzyme (SCCE) is coordinated in ovarian tumors. Int J Gynecol Cancer 200 I, I I (6):454-46I.

36. Tanimoto H, Underwood LJ, Shigemasa K, Yan Yan MS, Clarke J, Parmley TH, O'Brien TJ: The stratum corneum chymotryptic enzyme that mediates shedding and desquamation of skin cells is highly overexpressed in ovarian tumor cells. Cancer 1999, 86(10):2074-2082.

37. Tian X, Shigemasa K, Hirata E, Gu L, Uebaba Y, Nagai N, O'Brien T] Ohama K: Expression of human kallikrein 7 (hK7/SCCE) and its inhibitor antileukoprotease (ALP/SLPI) in uterine endocervical glands and in cervical adenocarcinomas. Oncol Rep 2004, I 2(5): 100I-1006.

38. Planque C, de Monte M, Guyetant S, Rollin J, Desmazes C, Panel V, Lemarie E, Courty Y: KLK5 and KLK7, two members of the human tissue kallikrein family, are differentially expressed in lung cancer. Biochem Biophys Res Commun 2005, 329(4): | 260- 266.

39. Koch PJ, Goldschmidt MD, Zimbelmann R, Troyanovsky R, Franke WW: Complexity and expression patterns of the desmosomal cadherins. Proc Natl Acad Sci USA 1992, 89(I):353-357.

40. Schafer S, Stumpp S, Franke WW: Immunological identification and characterization of the desmosomal cadherin Dsg2 in coupled and uncoupled epithelial cells and in human tissues. Differentiation 1996, 60(2):99-108.

4I. Mahoney MG, Simpson A, Aho S, Uitto J, Pulkkinen L: Interspecies conservation and differential expression of mouse desmoglein gene family. Exp Dermatol 2002, I I (2): I I5- I25.

42. Spindler V, Drenckhahn D, Zillikens D, Waschke J: Pemphigus IgG causes skin splitting in the presence of both desmoglein I and desmoglein 3. Am J Pathol 2007, I 7 I(3):906-916.

\section{Pre-publication history}

The pre-publication history for this paper can be accessed here:

http://www.biomedcentral.com/1471-2407/8/373/pre

pub

Publish with Bio Med Central and every scientist can read your work free of charge

"BioMed Central will be the most significant development for disseminating the results of biomedical research in our lifetime. "

Sir Paul Nurse, Cancer Research UK

Your research papers will be:

- available free of charge to the entire biomedical community

- peer reviewed and published immediately upon acceptance

- cited in PubMed and archived on PubMed Central

- yours - you keep the copyright
BioMedcentral 